\title{
INFLUENCE OF DENTAL BONDING SURFACE AND BONDING METHODS ON MARGINAL ADAPTATION OF OCCLUSAL VENEER AFTER THERMAL AGING
}

\author{
Lamia Dawood* and Walid Al-Zordk**
}

\begin{abstract}
Objectives: The aim of this study was to evaluate the influence of different dental bonding substrates and bonding methods on the marginal quality in mandibular molars restored using monolithic zirconia occlusal veneer after thermal aging.

Materials and Methods: Eighty extracted mandibular molars were collected and divided, based on type of dental bonding surface and bonding method, into eight groups. A non-retentive occlusal veneer preparation was performed to be either within enamel, dentin, dentin with intracoronal cavity, or dentin with composite filling. 1-mm zirconia occlusal veneers were fabricated. For each specimen, the marginal gap was measured (x40) using a stereoscope. Each occlusal veneer was bonded to its corresponding tooth using either etch-and-rinse or self-etch system. All specimens were thermal aged $\left(\$ 55^{\circ} \mathrm{C}\right)$ for 7500 cycles. The post-cementation marginal gap was measured. Two-way ANOVA test and post-hoc test were applied to test for significance in marginal gap values in relation to bonding substrates and bonding method.
\end{abstract}

Results: No statistically significant difference in pre-cementation marginal gap values was detected between different bonding surfaces. For post-cementation, two-way ANOVA test indicated significant differences in marginal gap values in relation to bonding substrates and bonding method.

Conclusions: The type of dental bonding surface (either enamel, dentin, dentin with composite filling, or dentin with intra-coronal cavity) can influence the marginal quality of zirconia occlusal veneers after thermal aging. Compared with self-etch, etch-and-rinse bonding method demonstrated superior marginal quality of zirconia occlusal veneer restoration after thermal aging.

KEYWORDS: Marginal accuracy, Occlusal tabletop, Ultra-thin, Total-etch, Self-etch.

\footnotetext{
* Associate Professor, Fixed Prosthodontics Dept., Faculty of Dentistry, Mansoura University, and Delta University for Science and Technology, Egypt.

** Associate Professor, Fixed Prosthodontics Dept., Faculty of Dentistry, Mansoura University, Egypt.
} 


\section{INTRODUCTION}

Depending on the severity of lesion, destruction of the occlusal enamel and exposure of the underlying dentin causing formation of occlusal defects as a consequence of pathological and functional problems such as tooth wear, erosion, or caries. ${ }^{1,2}$ The management of such lesions presents a challenge and could be treated with restorations such as inlays, onlays or crowns, ${ }^{3,4}$ however, these approaches can be very invasive. ${ }^{3}$ Therefore, with the continuous development of adhesive bonding methods, the minimally invasive approaches are recommended because it offers several advantages such as maximum preservation of sound tooth structure, maintain pulp vitality, and decrease postoperative sensitivity. ${ }^{5-7}$ Occlusal veneers, gained more popularity as a treatment modality for severely worn teeth. ${ }^{8}$ The extent of damage to severely worn teeth is irregular with defects in the occlusal surface. ${ }^{9}$ To restore these defects, the designs of the occlusal veneer preparation are modified..$^{10-14}$

The longevity of ceramic restorations is affected by many factors like type and thickness of ceramic material, occlusal forces, and quality of bonding. ${ }^{15,16}$ Furthermore, the type of bonding surfaces and the ability to gain long standing bond are contributing factors for the success of these restorations. ${ }^{17}$ However, teeth requiring occlusal veneer restoration seldomly have only single type of hard tooth substance to be restored. ${ }^{18}$ With occlusal veneers, the defective dental surfaces can vary greatly, and may be purely enamel defects, exposed dentin, or composite filling. ${ }^{19-21}$

Etch-and-rinse systems achieved successful bond to enamel. ${ }^{22}$ However, bond to exposed dentin was reported to have an adverse effect on the fracture strength of adhesively luted all-ceramic restorations. ${ }^{23}$ Therefore, self-etching primers are used to simplify the bonding procedure and enhance bonding to dentin, ${ }^{24}$ they appear to play an encouraging role to obtain long lasting bond to dentin. ${ }^{25}$ However, when using these primers on enamel, adhesive bonding is conceded compared to etching with phosphoric acid because of their limited etching ability. ${ }^{26}$ To improve the bond between the tooth structure and the restoration, selective etching of enamel areas are advised when using self-etching primers. ${ }^{25,27-29}$

The improved dental adhesive systems together with higher strength restorative dental materials used in minimal thicknesses, enabled minimal invasive replacement of lost hard tooth structures. ${ }^{30-32}$ Zirconium oxide ceramics have superior mechanical properties and have shown promising results as fixed restorations. ${ }^{34,35}$

The marginal accuracy is among the factors affecting the clinical outcomes of ceramic restorations..$^{36-40}$ Therefore, the purpose of this study was to evaluate the influence of different dental bonding substrates (enamel, dentin, dentin with intra-coronal cavity, and dentin with composite filling) and bonding methods (etch-and-rinse and self-etch) on the marginal quality in mandibular molars restored using monolithic zirconia occlusal veneer after thermal aging. The hypotheses tested were: (1) there is no significant difference in marginal quality between different dental bonding surfaces and (2) the bonding method has influence on the marginal quality of occlusal veneers.

\section{MATERIALS AND METHODS}

Eighty freshly extracted caries-free and intact human mandibular second molars were collected from the Oral and Maxillofacial Surgery Department, Faculty of Dentistry, Mansoura University. Teeth were extracted for periodontal reasons, from the age group of 45-60 years, and having average dimensions of $11 \pm 0.5 \mathrm{~mm}, 10.5 \pm 0.5 \mathrm{~mm}$, and $7.5 \pm 0.5 \mathrm{~mm}$ mesio-distal, bucco-lingual, and occluso-cervical, respectively. ${ }^{41}$ The selected teeth were free from crack, old restorations, or any defect. All included teeth were cleaned and stored in $0.1 \%$ thymol solution. Each tooth was vertically embedded in a self-curing acrylic resin material (Acrostone, cold cure, \#1659987, Egypt) with the cemento-enamel junction located $2 \mathrm{~mm}$ above the 
level of the resin. Using additional silicon impression material (Presigum, President Dental, Germany), a pre-preparation silicon index was fabricated for each tooth.

Study grouping: The eighty teeth were randomly divided into 8 groups according to the dental bonding substrate (Figure 1) and bonding method into: Group ES; occlusal veneer bonded to enamel using self-etch resin cement, Group ET; occlusal veneer bonded to enamel using etch-and-rinse resin cement, Group DS; occlusal veneer bonded to dentin using self-etch resin cement, Group DT; occlusal veneer bonded to dentin using etch-and-rinse resin cement, Group CS; occlusal veneer bonded to dentin with intra-coronal cavity using self-etch resin cement, Group CT; occlusal veneer bonded to dentin with intra-coronal cavity using etch-and-rinse resin cement, Group FS; occlusal veneer bonded to dentin with composite filling using self-etch resin cement, and Group FT; occlusal veneer bonded to dentin with composite filling using etch-and-rinse resin cement.

Tooth preparation: Tooth preparation was performed using a straight hand-piece (Traus ATII, Saeshin Precision Co., Korea) with the aid of a paralleling device (103 Surveyor, Marathon, China). All preparations were performed by a single operator (W.A.-Z), the occlusal veneer preparations were performed to follow the occlusal anatomy with $150^{\circ}$ divergence angle between cusps. For ES and ET groups, the occlusal surface was reduced by
$1 \mathrm{~mm}$ at cusp tips and central groove. For DS and DT groups, the occlusal surface was reduced by $2 \mathrm{~mm}$ ( $1 \mathrm{~mm}$ to simulate the occlusal wear and $1 \mathrm{~mm}$ for restoration). For CS and CT groups, after the occlusal surface was reduced by $2 \mathrm{~mm}$, the intra-coronal cavities were prepared using a diamond stone (856 blue, FG, Switzerland). The intra-coronal cavity had $1 \mathrm{~mm}$ pulpal depth, $2 \mathrm{~mm}$ bucco-lingual width, $1.6 \mathrm{~mm}$ away from the proximal marginal ridge, and 8-degree wall taper. For FS and FT groups, after the occlusal surface was reduced by $2 \mathrm{~mm}$ and the intra-coronal cavity was prepared, the intra-coronal cavities were also prepared as previously described, then a universal adhesive (Tertric N-Bond Universal, Ivoclar Vivadent, Liechtenstien) was applied over each intra-coronal cavity and light activated at each surface for 20 seconds following the manufacture's recommendations. Then, the intra-coronal cavities (FS and FT groups) were restored using a composite resin (Tetric N-Ceram Bulk-Fill, Ivoclar Vivadent, Liechtenstein) and light activated for 20 seconds. The pre-preparation silicon index was used to evaluate each preparation. Finally, all prepared teeth were smoothened and finished using tapered roundend diamond stone (856 yellow, FG, Switzerland). Polyvinyl siloxane impression (Betasil, MullerOmicron $\mathrm{GmbH} \&$ Co KG, Germany) was done for each specimen, then impressions were poured using type IV extra hard dental stone (Shera Premium, REF 101070-18, Shera Werkstoff-Technologie GmbH \& Co. KG, Germany).

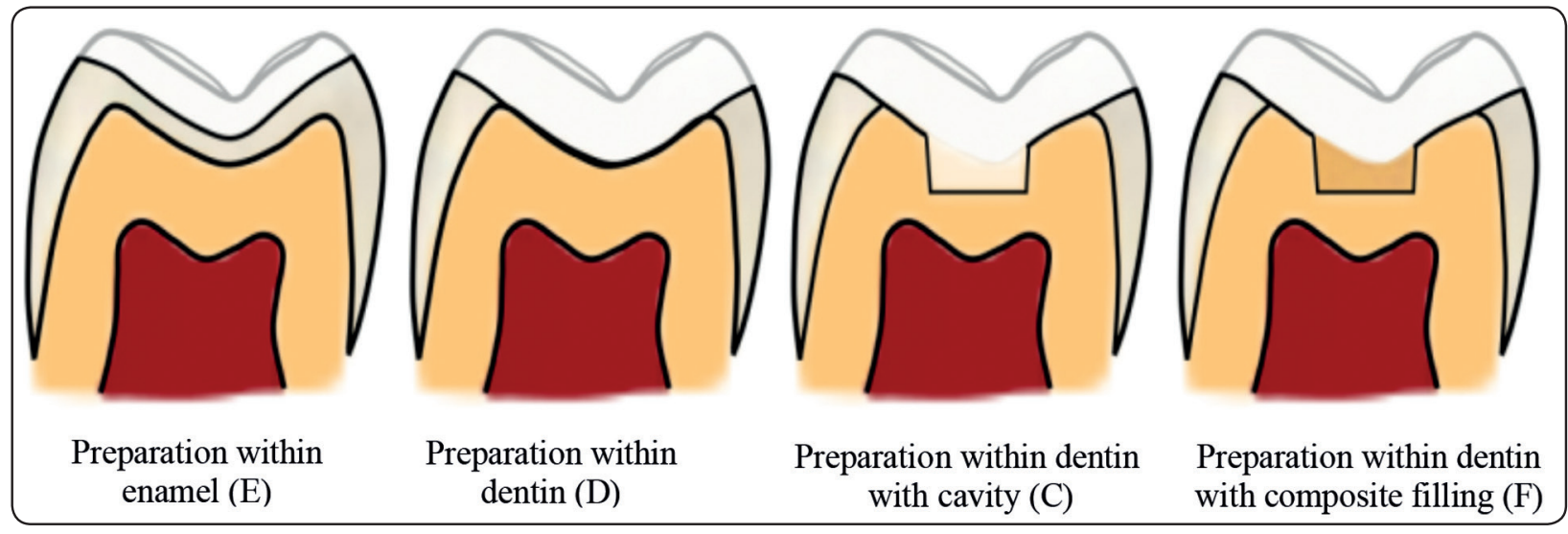

Fig. (1) Diagram represents the dental bonding surfaces for the study groups. 
Fabrication of occlusal veneers: Each die was scanned (ldentica Hybrid, Medit Corp., Seoul, Korea). Each veneer restoration was designed using CAD-CAM software (Valletta 2015 v2.2, Exocad $\mathrm{GmbH}$, Germany). The cement gap was set at 50 $\mu \mathrm{m}$. The thickness of all veneer restorations was $1 \mathrm{~mm}$ (except CS and CT groups at cavity projection areas). Then, the restorations were milled (Coritec 250i, Imes-Icore $\mathrm{GmbH}$, Germany) from zirconia block (Katana UTML Kuraray Noritake Dental Inc., Japan). After milling, zirconia restorations were sintered and glazed according to manufacturer's recommendations. Each restoration was carefully inspected to detect any cracks, then its seating was verified against its corresponding tooth.

Measurement of marginal gap before cementation: A stereoscope (Olympus SZ61, Tokyo, Japan) coupled with a camera (Tucsen $\mathrm{H}$ Series, China) and an image analysis software ( $\mathrm{T}$ Capture 5.1, Tucsen, China) was used (Figure 2). For each surface, the vertical marginal gap was measured (x40 magnification) at three points and the average was recorded.

Cementation: The intaglio surfaces of all zirconia occlusal veneers were air-borne particle abraded with $50 \mu \mathrm{m}$ alumina at a pressure of 2 bars from $10 \mathrm{~mm}$ distance. For ES, DS, CS, and FS groups, the zirconia veneers were treated with Ceramic Primer (Clearfil ceramic primer, Kuraray Dental Inc., Japan) and left undisturbed for 10 seconds and gently air-dried for 5 seconds. The tooth primer (Panavia V5 tooth primer, Kuraray
Dental Inc., Japan) was applied to prepared teeth and left for 20 seconds, then gently air-dried. The cement paste (Panavia V5 cement, Kuraray Dental Inc., Japan) was dispensed from auto-mix syringe into pretreated restoration, then seated over its corresponding tooth. Finally, the restoration was tack cured for 5 seconds and excess cement removed. Each surface of cemented restoration was light cured for 20 seconds. For ET, DT, CT, and FT groups, the ceramic primer (Z-Primer Plus, Bisco, IL, USA) was applied according to the recommendations of the manufacturer. Then, the peripheral enamel was selectively acid etched using 37\% Phosphoric acid (N-Etch, Ivoclar Vivadent, schaan, Liechtenstein). Then, the bonding agent (All-Bond Universal, Bisco, IL, USA) was applied and cured according to the recommendations of the manufacturer. Finally, the cement (Duo-Link Universal, Bisco, IL, USA) was applied to the intaglio surface of the restoration, then the restoration was seated to its corresponding tooth and held under a constant load of $10 \mathrm{~N} .{ }^{19}$ The light curing (Epilar 2500, 3M ESPE, USA) was performed for 40 seconds per each surface of the tooth. Then, finishing and polishing of the margins were done. All specimens were stored in water at 37 ${ }^{\circ} \mathrm{C}$ for two weeks. ${ }^{42}$

Aging: All specimens were subjected to thermal aging for 7,500 cycles (SD Mechatronics Thermocycler, Westerham, Germany) with temperature range from $5^{\circ} \mathrm{C}$ to $55^{\circ} \mathrm{C}$ and dwelling time of 30 seconds.

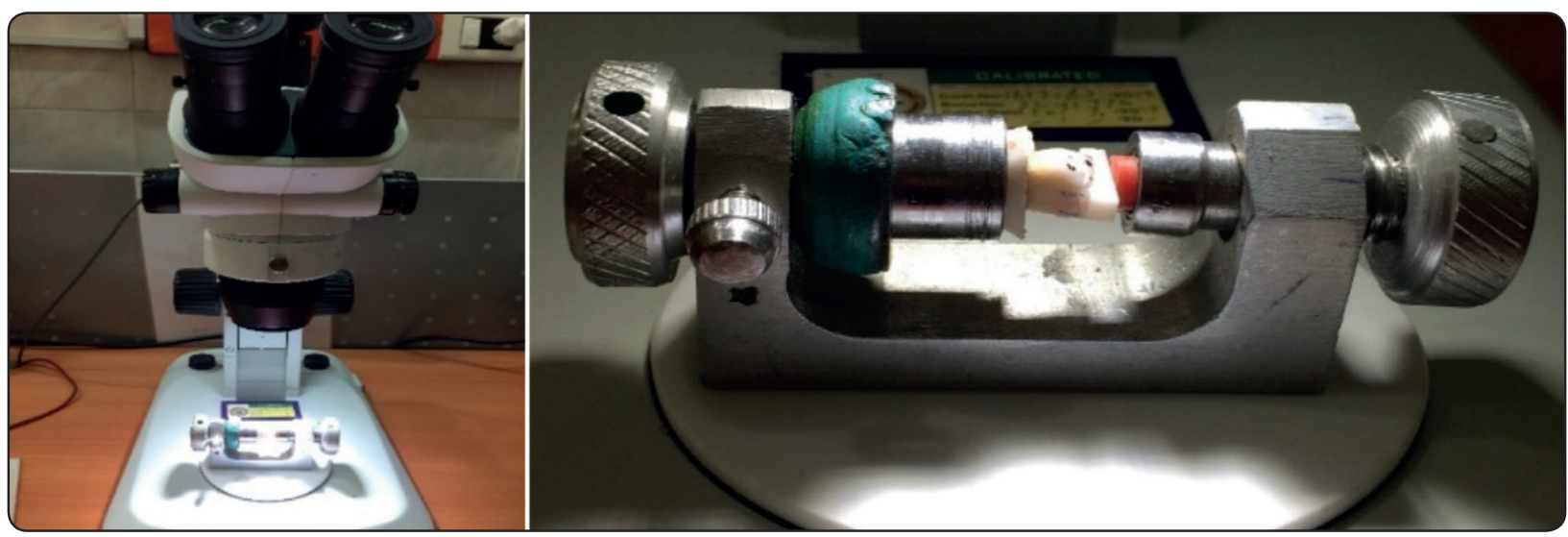

Fig. (2) Measurement of marginal gap using a specially designed holding device. 


\section{Measurement of marginal gap after cementation:}

For each restoration, the marginal gap was measured at the predetermined points as descried before.

\section{Statistical analysis:}

Data was analyzed using Statistical Package for Social Science software computer program version 26 (SPSS, Inc., Chicago, IL, USA). Oneway ANOVA test and tukey test were used for comparisons. Two-way ANOVA test followed by post-hoc Bonferroni were used to detect the effect of bonding method on different dental bonding surfaces. $\mathrm{P}$ value less than .05 was considered statistically significant.

\section{RESULTS}

For the pre-cementation marginal gap distance, means and standard deviations are presented in Table 1. One-way ANOVA test revealed no significant differences among groups.

Regarding the post-cementation marginal gap measurements, the means and standard deviations are presented in Table 2. One-way ANOVA and post-hoc Tukey tests revealed statistically significant difference between different dental substrate groups and $t$-test revealed statistically significant difference between the two bonding methods within each dental substrate group. For the interaction between the study variables, two-way ANOVA test (Table 3) revealed significant differences at buccal $(P=.011)$, lingual $(P<.001)$ and distal $(P=.001)$ surfaces, while there was no significant difference at mesial surface $(P=.554)$.

TABLE (1) The pre-cementation marginal gap $(\mu \mathrm{m})$ for bonding surfaces regarding the buccal, lingual, mesial, and distal surfaces.

\begin{tabular}{|c|c|c|c|c|}
\hline & Bonded to enamel & Bonded to dentin & $\begin{array}{c}\text { Bonded to dentin with intra- } \\
\text { coronal cavity }\end{array}$ & $\begin{array}{c}\text { Bonded to dentin with } \\
\text { composite filling }\end{array}$ \\
\hline Buccal & $46.33 \pm 4.93^{\mathrm{a}}$ & $52.33 \pm 5.09^{\mathrm{a}}$ & $54.67 \pm 4.93^{\mathrm{a}}$ & $54.33 \pm 6.09^{\mathrm{a}}$ \\
\hline Lingual & $45.67 \pm 1.37^{\mathrm{b}}$ & $49.33 \pm 2.73^{\mathrm{b}}$ & $47.00 \pm 4.98^{\mathrm{b}}$ & $48.33 \pm 1.86^{\mathrm{b}}$ \\
\hline Mesial & $47.00 \pm 3.58^{\mathrm{c}}$ & $49.33 \pm 5.75^{\mathrm{c}}$ & $52.67 \pm 6.59^{\mathrm{c}}$ & $46.67 \pm 4.50^{\mathrm{c}}$ \\
\hline Distal & $46.67 \pm 4.23^{\mathrm{d}}$ & $55.33 \pm 8.07^{\mathrm{d}}$ & $53.00 \pm 7.64^{\mathrm{d}}$ & $46.33 \pm 3.61^{\mathrm{d}}$ \\
\hline
\end{tabular}

Similar letters in the same row indicate non-significant differences $(P \leq .05)$

TABLE (2) The post-thermal aging marginal gap $(\mu \mathrm{m})$ for bonding surfaces regarding the buccal, lingual, mesial, and distal surfaces of occlusal veneers bonded using etch-and-rinse and self-etch resin cements.

\begin{tabular}{|l|c|c|c|c|c|}
\hline \multicolumn{2}{|c|}{} & Bonded to enamel & Bonded to dentin & $\begin{array}{c}\text { Bonded to dentin with } \\
\text { intra-coronal cavity }\end{array}$ & $\begin{array}{c}\text { Bonded to dentin with } \\
\text { composite filling }\end{array}$ \\
\hline \multirow{2}{*}{ Buccal } & $\mathrm{T}$ & $83.50 \pm 4.93^{\mathrm{a}}$ & $88.00 \pm 3.29^{\mathrm{a}}$ & $106.00 \pm 2.19^{\mathrm{b}}$ & $88.00 \pm 1.10^{\mathrm{a}}$ \\
\cline { 2 - 6 } & $\mathrm{S}$ & $97.50 \pm .55^{\mathrm{b}^{*}}$ & $94.50 \pm 6.02^{\mathrm{b}^{*}}$ & $122.00 \pm 3.29^{\mathrm{c}^{*}}$ & $95.00 \pm 6.57^{\mathrm{b}^{*}}$ \\
\hline \multirow{2}{*}{ Lingual } & $\mathrm{T}$ & $82.50 \pm 8.22^{\mathrm{d}}$ & $86.00 \pm 3.29^{\mathrm{d}}$ & $104.00 \pm 2.19^{\mathrm{e}}$ & $87.00 \pm 3.29^{\mathrm{d}}$ \\
\cline { 2 - 6 } & $\mathrm{S}$ & $98.00 \pm 9.86^{\mathrm{f}}$ & $100.00 \pm 6.57^{\mathrm{f}^{*}}$ & $121.00 \pm 12.05^{\mathrm{g}^{*}}$ & $99.50 \pm 3.83^{\mathrm{*}}$ \\
\hline \multirow{2}{*}{ Mesial } & $\mathrm{T}$ & $84.00 \pm 5.48^{\mathrm{h}}$ & $85.50 \pm 8.22^{\mathrm{h}}$ & $105.50 \pm 1.64^{\mathrm{i}}$ & $86.00 \pm 3.29^{\mathrm{h}}$ \\
\cline { 2 - 6 } & $\mathrm{S}$ & $96.50 \pm 4.93^{\mathrm{j}^{*}}$ & $100.00 \pm 2.19^{\mathrm{j}^{*}}$ & $119.50 \pm 2.74^{\mathrm{k}^{*}}$ & $95.50 \pm 4.93^{\mathrm{j}^{*}}$ \\
\hline \multirow{2}{*}{ Distal } & $\mathrm{T}$ & $87.50 \pm .55^{1}$ & $89.00 \pm 6.57^{1}$ & $101.50 \pm 2.74^{\mathrm{m}}$ & $86.50 \pm 2.74^{1}$ \\
\cline { 2 - 6 } & $\mathrm{S}$ & $99.00 \pm 5.48^{\mathrm{n}^{*}}$ & $99.00 \pm 1.10^{\mathrm{n}^{*}}$ & $123.00 \pm 4.38^{\mathrm{o}^{*}}$ & $96.50 \pm 2.74^{\mathrm{n}^{*}}$ \\
\hline
\end{tabular}

T: etch-and-rinse.

S: self-etch.

Different letters in the same row indicate significance $(P \leq .05)$

*: indicate significance between etch-and-rinse and self-etch within each group $(P \leq .05)$ 
TABLE (3) Two-way ANOVA test for the interaction between dental bonding substrates and bonding methods regarding the buccal, lingual, mesial and distal surfaces.

\begin{tabular}{|l|c|c|c|c|}
\hline & Buccal & Lingual & Mesial & Distal \\
\hline Bonding surfaces & $\mathrm{F}=95.835$ & $\mathrm{~F}=9212.846$ & $\mathrm{~F}=63.697$ & $\mathrm{~F}=77.973$ \\
& $P<.001^{*}$ & $P<.001$ & $P<.001$ & $P<.001$ \\
\hline Bonding method & $\mathrm{F}=86.207$ & $\mathrm{~F}=25.622$ & $\mathrm{~F}=89.014$ & $\mathrm{~F}=144.794$ \\
& $P<.001$ & $P<.001$ & $P<.001$ & $P<.001$ \\
\hline Bonding surfaces* & $\mathrm{F}=4.263$ & $\mathrm{~F}=52.983$ & $\mathrm{~F}=0.707$ & $\mathrm{~F}=6.340$ \\
Bonding method & $P=.011$ & $P<.001$ & $P=.554$ & $P=.001$ \\
\hline
\end{tabular}

\section{DISCUSSION}

The null hypotheses that the tested types of dental bonding surfaces would not affect the marginal quality, and the bonding method has influence on the marginal quality of occlusal veneers were partially accepted because the marginal quality of occlusal veneers is influenced by both the type of dental bonding surface and the bonding method.

In the present study, human molar teeth with similar dimensions were selected and standardized tooth reparation was performed. Almost all the occlusal veneer preparations were performed in the dentin to simulate the advanced clinical wear. ${ }^{8} \mathrm{~A}$ ceramic thickness of $1 \mathrm{~mm}$ was chosen to preserve as much tooth structure as possible. It was reported that the thickness of occlusal veneer restorations must not fall below 0.7 to $1 \mathrm{~mm}$ regardless of the material type..$^{13}$ Other studies considered the thickness of $0.8 \mathrm{~mm}$ as the appropriate threshold for lithium disilicate occlusal veneers. ${ }^{18}$ However, it was reported that when the potential space for restoration differs, alternative occlusal veneer materials should be appraised..$^{14}$ In the current study, occlusal veneers were fabricated using CAD-CAM technology which allow precise control on thickness and anatomy during fabrication process.

Additionally, the occlusal veneer preparation was limited to the occlusal surface only without finish line extended over the axial walls. Occlusal veneers, thin overlay restorations with a non- retentive design, represent a conservative treatment modality in comparison with traditional onlays and complete-coverage crowns. ${ }^{5}$ Occlusal veneer covering only the occlusal surface created minimal stresses within the restoration. ${ }^{10,11}$ Clausen et $\mathrm{al}^{20}$ found that the fracture strength of occlusal veneers was not affected by the presence of a finish line, also it was not influenced by type of preparation design either in enamel or in dentin. Additionally, Zhang et $\mathrm{al}^{14}$ studied the occlusal veneer designs and found that stress bearing areas were generated at the cusp tips under thin occlusal veneer when prepared with finish line over the axial walls. Additionally, Huang et $\mathrm{al}^{10}$ found that occlusal veneers which covered just the occlusal surface showed decrease in maximum stresses in the restoration and high fracture resistance than traditional full coverage crown and occlusal veneers which partially covered the axial surfaces. In the current study, the preparation design was chosen based on studies of Clausen et $\mathrm{al}^{20}$, Sasse et $\mathrm{al}^{18}$ and based on general guidelines of preparation. ${ }^{43}$

In the current study, the cement thickness was set to be $50 \mu \mathrm{m}$. There is no consensus in the literature about the fit values of indirect restorations and the diversity could be attributed to the testing method, testing parameters and ceramic system investigated. ${ }^{36}$ It was reported that marginal gap less than $80 \mu \mathrm{m}$ is acceptable, while other studies considered $100 \mu \mathrm{m}$ up to $200 \mu \mathrm{m}$ as acceptable misfit values. ${ }^{37}$ 
Several methods are used to analyse and evaluate the marginal and internal gap and the fit of the restorations..$^{38}$ In the current study, vertical marginal gap was measured using direct examination under stereomicroscope since it was an easy, rapid and convenient method for measuring. Additionally, evaluation before and after cementation allowed a more understanding of the role of cementation process. In the present study, there was no significant differences between different bonding substrates before cementation. Angerame et $\mathrm{al}^{21}$ found no significant differences in margin quality with two preparation designs at time zero.

The results of the current study showed elevation in mean values of the marginal gap after cementation and thermal aging. It was reported that oral fluids degrade the resin matrix causing the marginal adaptation of bonded restoration to breakdown. ${ }^{44}$ Thermal stresses occur at the adhesive interface as a result of different thermal expansion coefficients of the materials which can induce alterations in the materials. ${ }^{45}$ The thermal expansion coefficients of enamel and dentin are about $17 \times 10^{-6} /{ }^{\circ} \mathrm{C}$ and $11 \times 10^{-6} /{ }^{\circ} \mathrm{C}$, respectively, while the thermal expansion coefficients of adhesive and resin cements lie between $20 \times 10^{-}$ $6 /{ }^{\circ} \mathrm{C}$ to $80 \times 10^{-6} /{ }^{\circ} \mathrm{C} .{ }^{46}$ Additionally, the problem is amplified with presence of composite filling which may exhibit high thermal expansion coefficient. ${ }^{47}$ In the current study, specimens were thermal cycled to simulate the physical stresses in the oral cavity. The thermal stresses occur at the bonding interface. ${ }^{48}$ Furthermore, the marginal discrepancy could be affected by the hydrolytic pressure of the luting cement. ${ }^{39}$ However, Angerame et $\mathrm{al}^{21}$ studied the marginal adaptation for two preparation designs for occlusal veneers and found that no differences in the marginal quality. Their results could be attributed to the designs tested which are extended to the axial walls. Another study reported a significantly decreased marginal quality after aging for specimens bonded to enamel using self- etch system. ${ }^{28}$ Regarding the presence of composite filling as a bonding substrate, Müller et $\mathrm{al}^{29}$ found that ceramic inlays bonded to composite showed marginal sealing comparable to bonding to dentin.

In the current results, there were significant differences when bonding using etch-and-rinse and self-etch systems. This could be related to the higher viscosity of self-etch cement compared with that of etch-and-rinse. ${ }^{30}$ A study found that the self-etch resin cement revealed better marginal adaptation compared to self-adhesive cements. This may be related to the use of ultrasonic scaling at the restoration margins. ${ }^{40}$ Regarding the interaction between the study variables, there was no significant difference with the mesial marginal gap which may be attributed to what is called overshoot phenomena that simulate virtual peaks near the edges. ${ }^{32}$

As a limitation to the current study, only one thickness of non-retentive occlusal veneers was studied, thus variable thicknesses need to be evaluated because there are more thickness variations in clinical application. Also, other designs together with different types of resin cements need to be evaluated with respect to different dental bonding surfaces. Furthermore, clinical studies are required to predict the clinical performance of occlusal veneers.

\section{CONCLUSIONS}

Under the limitations of this study, the following conclusions could be drawn:

1. The type of dental bonding surface (either enamel, dentin, dentin with composite filling, or dentin with intra-coronal cavity) can influence the marginal quality of zirconia occlusal veneers after thermal aging.

2. Compared with self-etch, etch-and-rinse bonding method demonstrated superior marginal quality of zirconia occlusal veneer restoration after thermal aging. 


\section{REFERENCES}

1. Lussi A, Jaeggi T. Erosion--diagnosis and risk factors. Clin Oral Investig. 2008;12:S5-13.

2. Resende T, Reis K, Schlichting L, Magne P. Ultrathin CAD-CAM ceramic occlusal veneers and anterior bilaminar veneers for treatment of moderate dental biocorrosion: a 1.5-year follow-up. Oper Dent. 2018;43:337-346.

3. Vailati F, Belser UC. Full-mouth adhesive rehabilitation of a severely eroded dentition: the three-step technique. part 1. Eur J Esthet Dent. 2008;3:30-44.

4. Freitas AC, Jr., Silva AM, Lima Verde MA, Jorge de Aguiar JR. Oral rehabilitation of severely worn dentition using an overlay for immediate re-establishment of occlusal vertical dimension. Gerodontology. 2012;29:75-80

5. Rocca GT, Rizcalla N, Krejci I, Dietschi D. Evidencebased concepts and procedures for bonded inlays and onlays. Part II. Guidelines for cavity preparation and restoration fabrication. Int J Esthet Dent. 2015;10:392-413

6. Cardoso MV, de Almeida Neves A, Mine A, Coutinho E, Van Landuyt K, De Munck J, al e. Current aspects on bonding effectiveness and stability in adhesive dentistry. Aust Dent J. 2011;56:31-44

7. Walter R, Swift EJ, Jr., Boushell LW, Braswell K. Enamel and dentin bond strengths of a new self-etch adhesive system. J Esthet Restor Dent. 2011;23:390-396

8. Maeder M, Pasic P, Ender A, Ozcan M, Benic G, Ioannidis A. Load-bearing capacities of ultra-thin occlusal veneers bonded to dentin. J Mech Behav Bio Mater. 2019;95:165-171.

9. A. Lussi, E. Hellwig, C. Ganss, T. Jaeggi, Buonocore memorial lecture, Dental erosion, Oper Dent. 2009;34:251262.

10. Huang X, Zou L, Yau R, Wu S, Li Y. Effect of preparation design on the fracture behavior of ceramic occlusal veneers in maxillary premolars. J Dent. 2020;97:103346.

11. Veneziani M. Posterior indirect adhesive restorations: updated indications and the morphology driven preparation technique. Int J Esthet Dent. 2017;12:204-230

12. Lierop J, Moodley D, Mulder R. Influence of ceramic thickness and cavity design optimization on fracture resistance of partial coverage restorations. The New Zealand dent J. 2019;115:13-19.

13. Magne P, Schlichting LH, Maia HP, Baratieri LN. In vitro fatigue resistance of $\mathrm{CAD} / \mathrm{CAM}$ composite resin and ceramic posterior occlusal veneers. J Prosthet Dent. 2010;104:149-157
14. Zhang H, Lv P, Du W, Jiang T. Comparison of Fracture Load and Surface Wear of Microhybrid Composite and Ceramic Occlusal Veneers. J Prosthodont. 2020;29:387-393

15. Al-Akhali M, Chaar MS, Elsayed A, Samran A, Kern M. Fracture resistance of ceramic and polymer-based occlusal veneer restorations. J Mech Behav Biomed Mater. 2017;74:245-250.

16. Magne P. IDS: Immediate Dentin Sealing (IDS) for tooth preparations. J Adhes Dent. 2014;16:594. doi: 10.3290/j. jad.a33324.

17. Malament KA, Socransky SS. Survival of Dicor glass-ceramic dental restorations over 16 years. Part III: effect of luting agent and tooth or tooth-substitute core structure. J Prosthet Dent. 2001;86:511-9.

18. Sasse M, Krummel A, Klosa K, Kern M. Influence of restoration thickness and dental bonding surface on the fracture resistance of full-coverage occlusal veneers made from lithium disilicate ceramic. Dent Mater. 2015;31:907-915.

19. Krummel A, Garling A, Sasse M, Kern M. Influence of bonding surface and bonding methods on the fracture resistance and survival rate of full-coverage occlusal veneers made from lithium disilicate ceramic after cyclic loading. Dent Mater. 2019;35:1351-1359.

20. Clausen JO, Abou Tara M, Kern M. Dynamic fatigue and fracture resistance of non-retentive all-ceramic full-coverage molar restorations. Influence of ceramic material and preparation design. Dent Mater. 2010;26:533-538

21. Angerame D, De Biasi M, Agostinetto M, Franzò A, Marchesi G. Influence of preparation designs on marginal adaptation and failure load of full-coverage occlusal veneers after thermomechanical aging simulation. J Esthet Restor Dent. 2019;31:280-289.

22. Peumans M, Kanumilli P, De Munck J, Van Landuyt K,Lambrechts P, Van Meerbeek B. Clinical effectiveness ofcontemporary adhesives: a systematic review of currentclinical trials. Dent Mater. 2005;21:864-881.

23. Burke FJ. Survival rates for porcelain laminate veneers withspecial reference to the effect of preparation in dentin: aliterature review. J Esthet Restor Dent. 2012;24:257-265.

24. De Munck J, Van Landuyt K, Peumans M, Poitevin A,Lambrechts P, Braem M, et al. A critical review of thedurability of adhesion to tooth tissue: methods and results. J Dent Res. 2005;84:118-132.

25. Frankenberger R, Lohbauer U, Roggendorf MJ, Naumann M,Taschner M. Selective enamel etching reconsidered: betterthan etch-and-rinse and self-etch? J Adhes Dent. 2008;10:339-344. 
26. Hikita K, Van Meerbeek B, De Munck J, Ikeda T, Van LanduytK, Maida T, et al. Bonding effectiveness of adhesive lutingagents to enamel and dentin. Dent Mater. 2007; 23:71-80.

27. Federlin M, Hiller KA, Schmalz G. Effect of selective enameletching on clinical performance of CAD/CAM partial ceramiccrowns luted with a self-adhesive resin cement. Clin OralInvest. 2014;18:1975-1984.

28. Frankenberger R, Lohbauer U, Schaible RB, Nikolaenko SA,Naumann M. Luting of ceramic inlays in vitro: marginalquality of self-etch and etch-and-rinse adhesives versusself-etch cements. Dent Mater. 2008;24:185-191.

29. Müller V, Friedl KH, Friedl K, Hahnel S, Handel G, Lang R.Influence of proximal box elevation technique on marginalintegrity of adhesively luted Cerec inlays. Clin Oral Investig. 2017;21:607-612.

30. Zeller DK, Jens Fischer J, Rohr N. Viscous behavior of resin composite cements. Dent Mater J. 2020; doi:10.4012/ dmj.2019-313 JOI JST.JSTAGE/dmj/2019-313.

31. Edelhoff D, Sorensen JA. Tooth structure removal associated with various preparation designs for posterior teeth. Int J Periodontics Restorative Dent. 2002;22:241-249.

32. Marcia B, Paulo F, Jason G, Alvaro B. Adaptation of all-ceramic fixed partial dentures. Dent Mater. 2012;27:1119-1126.

33. Nakamura K, Harada A, Inagaki R, Kanno T, Niwano Y, Milleding P, et al. Fracture resistance of monolithic zirconia molar crowns with reduced thickness. Acta Odontol Scand. 2015;73:602-608

34. Weigl P, Sander A. In-vitro performance and fracture strength of thin monolithic zirconia crowns. J Adv Prosthodont. 2018;10:79-84

35. Vieira A, dos Santos M, Antunes L, et al. time and sealing effect of cavities prepared by an ultrasonic device and highspeed diamond rotator cutting system. J Oral Sci. 2007;49:207-211.

36. Korkut L, Cotert HS, Kurtulmus H. Marginal, internal fit and microleakage of zirconia infrastructures: an in-vitro study. Oper Dent. 2011;36:72-79.
37. Holmes R, Bayne C, Holland A, Sulik D. Considerations in measurments of marginal fit. J Prosthet Dent. 1989;62:405408.

38. Demir N, Ozturk A, Malkoc M. Evaluation of the marginal fit of full ceramic crowns by the microcomputed tomography (micro-CT) technique. Eur J Dent. 2014;8:437-444.

39. Azer AS. The effect of ultrasonic scaling on microleakage around zirconia crown margins luted with two resin cements. EDJ. 2020;66:2481-2489.

40. Nelson SJ, Ash Jr M M. The permanent mandibular molars. In Wheeler's dental anatomy, physiology, and occlusion 9th Ed, Saunders Elsevier Inc. 2010: P190.

41. Yazigi C, Schneider H, Chaar MS, Rüger C, Haak R, Kern M. Effects of artificial aging and progression of cracks on thin occlusal veneers using SD-OCT. J Mech Behav Biomed Mater. 2018 Dec;88:231-237.

42. Frankenberger R, Mörig G, Blunck U, Hajtó J, Pröbster L,Ahlers MO. Guidelines on the preparation for all-ceramicinlays and partial crowns - with special regard toCAD/ CAM-technology. Teamwork 2007;10:86-92.

43. Roulet JF, Walti C. Influence of oral fluid on composite resin and glass-ionomer cement. J Prosthet Dent. 1984; 52:182-189.

44. Oyafuso DK, Ozcan M, Bottino MA, Itinoche MK. Influence of thermal and mechanical cycling on the flexural strength of ceramics with titanium or gold alloy frameworks. Dent Mater. 2008;24:351-356.

45. Anusavice KJ, Brantley WA. Physical properties of dental materials. In: Anusavice KJ, ed. Phillips' Science of Dental Materials. 11th ed. St. Louis, MO: Saunders; 2005:52-53.

46. Versluis A, Douglas WH, Sakaguchi RL. Thermal expansion coefficient of dental composites measured with strain gauges. Dent Mater. 1996;12:290-294.

47. Pfeiffer P, Marx R. Temperature loading of resinbondedbridges and its effect on the composite strength of theadhesive bond. Schweiz Monatsschr Zahnmed 1989;99:782-786. 